\title{
Possible Dynamical Mechanisms for Southern Hemisphere Climate Change due to the Ozone Hole
}

\author{
Andrew OrR, Thomas J. Bracegirdle, And J. ScotT Hosking \\ British Antarctic Survey, Cambridge, United Kingdom \\ THOMAS JUNG* \\ European Centre for Medium-Range Weather Forecasts, Reading, United Kingdom \\ JOANNA D. HAIGH \\ Imperial College London, London, United Kingdom \\ TONY PHILLIPS \\ British Antarctic Survey, Cambridge, United Kingdom \\ WUHU FENG \\ University of Leeds, Leeds, United Kingdom
}

(Manuscript received 11 August 2011, in final form 24 April 2012)

\begin{abstract}
The authors report a hypothesis for the dynamical mechanisms responsible for the strengthening of the Southern Hemisphere circumpolar winds from the lower stratosphere to the surface due to the ozone hole. A general circulation model forced by stratospheric ozone depletion representative of the ozone hole period successfully reproduced these observed changes. Investigation of the dynamical characteristics of the model therefore provides some insight into the actual mechanisms. From this the authors suggest the following: 1 ) An initial (radiative) strengthening of the lower-stratospheric winds as a result of ozone depletion conditions the polar vortex so that fewer planetary waves propagate up from the troposphere, resulting in weaker planetary wave driving. 2) This causes further strengthening of the vortex, which results in an additional reduction in upward-propagating planetary waves and initiates a positive feedback mechanism in which the weaker wave driving and the associated strengthened winds are drawn downward to the tropopause. 3) In the troposphere the midlatitude jet shifts poleward in association with increases in the synoptic wave fluxes of heat and momentum, which are the result of a positive feedback mechanism consisting of two components: 4) increases in low-level baroclinicity, and the subsequent generation of baroclinic activity (associated with a poleward heat flux), are collocated with the jet latitudinal position, and 5) strengthening anticyclonic shear increases the refraction of wave activity equatorward (associated with a poleward momentum flux). Finally, 6) confinement of planetary waves in the high-latitude troposphere is an important step to couple the stratospheric changes to the tropospheric response.
\end{abstract}

* Current affiliation: Alfred Wegener Institute for Polar and Marine Research (AWI), Bremerhaven, Germany.

Corresponding author address: Dr. Andrew Orr, British Antarctic Survey, High Cross, Madingley Road, Cambridge CB3 0ET, United Kingdom.

E-mail: anmcr@bas.ac.uk

\section{Introduction}

Observations (e.g., Thompson and Solomon 2002, 2005; Thompson et al. 2011) and numerical models (e.g., Gillett and Thompson 2003; Shindell and Schmidt 2004; Arblaster and Meehl 2006; Perlwitz et al. 2008; Son et al. 2009, 2010; Polvani et al. 2011a) suggest that Antarctic stratospheric ozone depletion not only impacts the 
circulation of the stratosphere but also has an influence extending into the troposphere. Anomalies in the strength of the Southern Hemisphere ( $\mathrm{SH})$ stratospheric polar vortex peak during late spring, preceding similarly signed anomalies in the tropospheric circulation during summer.

The stratospheric changes are broadly consistent with the direct radiative impact of the formation of the Antarctic ozone hole, where about half of the total column ozone is depleted each spring (Solomon et al. 2005). Ozone absorbs incoming solar radiation and hence ozone depletion leads to a cooling of the polar stratosphere (e.g., Randel and Wu 1999). To maintain thermal wind balance, the resulting increasing meridional temperature gradient must be accompanied by changes in the vertical shear of the zonal wind, leading to a strengthening of the stratospheric polar vortex.

In the troposphere the anomalous circulation is characterized by low geopotential heights over Antarctica accompanied by higher geopotential heights over $\mathrm{SH}$ midlatitudes. This pattern of change is reflected in a trend toward a more positive phase of the southern annular mode (SAM) index (e.g., Thompson and Solomon 2002; Marshall 2003; Fogt et al. 2009), which is the dominant mode of variability of the $\mathrm{SH}$ extratropical circulation and describes the north-south shift of the midlatitude zonal jet about its climatological state between high $\left(50^{\circ}-60^{\circ} \mathrm{S}\right)$ and low $\left(30^{\circ}-40^{\circ} \mathrm{S}\right)$ latitudes (e.g., Karoly 1990; Hartmann and Lo 1998; Thompson and Wallace 2000). During the positive SAM phase the midlatitude jet is displaced poleward to high latitudes. Consequently, over high latitudes there has been a marked strengthening of the prevailing eastward surface winds that encircle Antarctica, as well as concomitant weakening of surface winds at $\mathrm{SH}$ midlatitudes. The anomalous zonal winds associated with such vacillations have a barotropic (i.e., constant with height), nearly zonally symmetric, vertical structure. These trends have had a significant impact on the regional climate of the $\mathrm{SH}$ [Thompson et al. (2011) and references therein].

The SAM is an internal or natural mode of unforced variability; that is, it is driven by dynamical processes internal to the atmosphere (e.g., Robinson 1991; Limpasuvan and Hartmann 1999, 2000). The persistent zonal flow anomalies associated with such annular variability result from a positive feedback between zonal mean wind anomalies and high-frequency eddies (period less than 10 days) associated with synoptic-scale baroclinically unstable disturbances (e.g., Karoly 1990; Robinson 1991; Yu and Hartmann 1993; Feldstein and Lee 1998; Hartmann and Lo 1998; Limpasuvan and Hartmann 1999, 2000; Lorenz and Hartmann 2001). These disturbances are associated with eddy fluxes of heat in the lower troposphere and momentum in the upper troposphere, and draw their energy from the available potential energy associated with large horizontal temperature gradients (i.e., baroclinic instability). The time mean of these eddies defines the so-called storm tracks, which are collocated with the latitude of the midlatitude jet.

During the positive SAM phase the proposed positive feedback between the eddies and the low-frequency flow is as follows: the strength of the lower-level winds of eastward barotropic anomalies in zonal wind structure is reduced by drag, resulting in eastward shear with height that must be accompanied by an increased horizontal temperature gradient (i.e., anomalous low-level baroclinicity coincides with the latitude of the anomalous midlatitude jet). Baroclinic eddies are generated more vigorously in such a region, equated with anomalously strong poleward eddy heat fluxes in the lower troposphere. This eddy activity tends to be refracted equatorward, consistent with the behavior of LC1 or "anticyclonic" baroclinic life cycles (Thorncroft et al. 1993), causing a poleward momentum flux convergence that acts to accelerate the zonal wind on the jet's poleward side (as well as decelerating the zonal wind on the jet's equatorward side). This reinforces the barotropic anomaly in zonal wind structure and the associated anticyclonic shear, and thus strengthens the eastward shear and the accompanying baroclinic generation of eddy activity (e.g., Robinson 1996, 2000; Lorenz and Hartmann 2001; Barnes and Hartmann 2010) and its subsequent refraction equatorward (Simpson et al. 2009, 2012), which together enable the jet to maintain its anomalous poleward displacement. Robinson (1996) showed that the persistence of the eddy fluxes significantly decreased if the horizontally sheared barotropic component of the flow was suppressed, suggesting that organization of the eddies by this component is also important. In addition, many studies argue that active feedback between the baroclinic eddy life cycle and barotropic shear results in the eddies acting to enhance the initial zonal flow anomalies (e.g., Yu and Hartmann 1993; Hartmann 1995; Hartmann and Lo 1998).

There is a clear lag between the tropospheric circulation response to Antarctic ozone depletion and the stratospheric circulation response. The vertical structure of circulation anomalies through late spring and early summer shows downward propagation of the anomalously strong stratospheric vortex to the tropopause over the course of some weeks, and thereafter a rapid descent through the troposphere over the course of a few days, with the troposphere anomalies persisting (Thompson and Solomon 2002, 2005; Gillett and Thompson 2003; Arblaster and Meehl 2006; Perlwitz et al. 2008; Thompson et al. 2011). There are many other studies that also show 
that variations in the $\mathrm{SH}$ stratospheric flow propagate downward and affect the troposphere, implying a direct effect of anomalies in the stratospheric circulation on the troposphere (e.g., Kuroda and Kodera 1998; Polvani and Kushner 2002; Graversen and Christiansen 2003; Thompson et al. 2005). Realistic simulation and understanding of these changes is important. Yet despite these studies, the mechanisms by which the stratospheric ozone hole influences the $\mathrm{SH}$ climate remain unclear (e.g., Hartmann et al. 2000; Gillett and Thompson 2003; Son et al. 2008; McLandress et al. 2010; Thompson et al. 2011).

The downward propagation of zonal mean zonal wind anomalies in the stratosphere are closely associated with anomalies in stratospheric wave drag caused by the vertical extent of the propagation of planetary-scale waves (e.g., Kuroda and Kodera 1999; Baldwin and Dunkerton 2001; Christiansen 2001), which is determined by the strength and structure of the stratospheric vortex, with a strong vortex associated with weak upward wave propagation (Kushner and Polvani 2004; Polvani and Waugh 2004). This depends on the fact that the upward propagation of planetary waves from the troposphere to the stratosphere is sensitive to the vertical shear of the zonal wind as well as the vertical gradient of temperature near the tropopause (Chen and Robinson 1992). This can initiate a positive feedback in which an anomalously strong vortex and associated increased vertical shear results in waves penetrating to successively lower altitudes, so that wind and wave driving anomalies are together drawn downward toward the tropopause (Kuroda and Kodera 1999; Baldwin and Dunkerton 2001; Christiansen 2001).

As the annular modes are fundamentally a result of internal dynamical feedback, they may respond significantly to rather modest external forcing. Previous studies have emphasized the influence of stratospheric forcing on the synoptic-scale wave fluxes of heat and momentum which drive the tropospheric annular modes and the requirement for eddy-zonal flow feedbacks. For example, increasing the horizontal temperature gradient in the lower stratosphere (i.e., increasing the shear and strengthening the vortex) changes these fluxes in such a way as to drive the midlatitude jet poleward to high latitudes (e.g., Hartmann et al. 2000; Kushner and Polvani 2004; Song and Robinson 2004; Haigh et al. 2005; Wittman et al. 2007; Simpson et al. 2009). Hartmann et al. (2000) and Thompson et al. (2011) suggest that this could potentially be the method by which the effects of stratospheric ozone depletion influence the $\mathrm{SH}$ tropospheric circulation.

SH tropospheric winds, and the climate in regions that they influence, can be expected to change in the first half of the twenty-first century as the Antarctic ozone hole gradually disappears with the continued implementation of the Montreal Protocol. Chemistry-climate models (CCMs), which have a fully interactive stratospheric chemistry, predict that this increase in spring stratospheric ozone will result in an equatorward displacement of the midlatitude jet during summer (e.g., Perlwitz et al. 2008; Son et al. 2008), although this impact will be opposed by continued increase of greenhouse gases (e.g., Shindell and Schmidt 2004; Polvani et al. 2011b). A further consequence of the marked strengthening of the lowerstratospheric polar vortex in recent decades has been an increasing delay in the breakup of the vortex, with the transition from eastward winds characteristic of winter to westward winds characteristic of summer occurring 20-30 days later in the 1990s than in the 1960s (Haigh and Roscoe 2009). However, in the majority of CCM simulations this transition is even more delayed (i.e., later than observed breakup) (e.g., Eyring et al. 2006). Improved simulation and understanding of these changes would also be aided by clarifying the mechanisms by which the ozone hole influences the SH climate.

The purpose of this paper is to use a transformed Eulerian mean (TEM) momentum budget analysis to investigate the hypothesis that stratospheric ozone depletion influences the circulation of the stratosphere and the troposphere as a result of changes to wave propagation that are supported by strong dynamical feedbacks (as reviewed above). Here, we realistically model the response to this perturbation by forcing a state-of-theart atmospheric model with prescribed stratospheric ozone depletion. Through investigation of the dynamical characteristics of the model, we suggest that a feedback mechanism causing fewer planetary waves to propagate up from the troposphere into the stratosphere, resulting in a decrease in the wave-driven deceleration of the polar vortex, is important in driving the downward propagation in the lower stratosphere. We further suggest that increases in the synoptic-scale wave fluxes of heat and momentum that drive the annular modes are important in driving the tropospheric response. Furthermore, our results suggest that planetary wave deceleration in the high-latitude upper troposphere is an important step in coupling the stratospheric changes to the tropospheric response. We demonstrate that the zonal wind forcing induced by such processes is in good quantitative agreement with the actual zonal wind tendency. It is stressed that this is not proof of a causal mechanism, but the results are consistent with our hypothesis, which allows us to make a suggestive statement of the importance of these mechanisms. The model, data, and method of analysis are discussed in section 2 . Section 3 presents the results. The paper finishes with a summary and discussion in section 4 . 


\section{Model, data, and method of analysis}

\section{a. Model}

The model used is the atmosphere-only component of the Hadley Centre Global Environmental Model version 3 (HadGEM3-A) (Martin et al. 2006). The horizontal resolution is $1.25^{\circ} \times 1.875^{\circ}$, with 85 vertical levels, from the surface up to around $85 \mathrm{~km}$, giving the high resolution in the stratosphere required to capture downward propagation of circulation anomalies (Karpechko et al. 2008). A control simulation forced by a seasonally varying distribution of ozone representative of the preozone hole period (i.e., preindustrial), and a perturbed simulation forced by a seasonally varying distribution of ozone representative of the ozone hole period (i.e., averaged over the period 19902000), were run for $24 \mathrm{yr}$ following $6 \mathrm{yr}$ of model spinup period. Both simulations were forced at the surface by the same seasonally varying, climatological sea surface temperatures and sea ice conditions generated by averaging Atmospheric Model Intercomparison Project version 2 (AMIP2) data over the period 1979-2006, which are time interpolated by the model to obtain the daily values. The ozone climatologies were obtained from the Atmospheric Chemistry and Climate (AC\&C) Stratospheric Processes and Their Role in Climate (SPARC) ozone database, which was created in support of the World Climate Research Programme's (WRCP's) Coupled Model Intercomparison Project phase 5 (CMIP5) (Cionni et al. 2011). Figure 1 displays the difference between the ozone hole and preozone hole climatologies at $70^{\circ} \mathrm{S}$. The Antarctic ozone hole can be seen clearly in the lower stratosphere during the $\mathrm{SH}$ spring, with the maximum difference in ozone reaching $70 \%$ in October at an altitude of about $100 \mathrm{hPa}$. The response of the model to this prescribed stratospheric ozone loss is evaluated by examining the 24-yr averaged differences between the two simulations. Results are presented as four 15-day averages starting on 2 November (i.e., late spring) and finishing on 1 January (i.e., early summer). The total 60-day period encompasses the onset (2-16 November), growth (17 November-1 December), decline (2-16 December), and decay (17 December-1 January) of the lower-stratospheric circulation response described above (Limpasuvan et al. 2004). (Note that the model uses a 360-day calendar year, with each year encompassing 12 months of 30 days each.) The temperature and wind fields were archived every $6 \mathrm{~h}$ at 18 vertical pressure levels: 1000, 950, 925, 850, 700, 600, 500, 400, $300,250,200,150,100,70,50,30,20$, and $10 \mathrm{hPa}$. All 15-day averages were computed from the 6-hourly data.

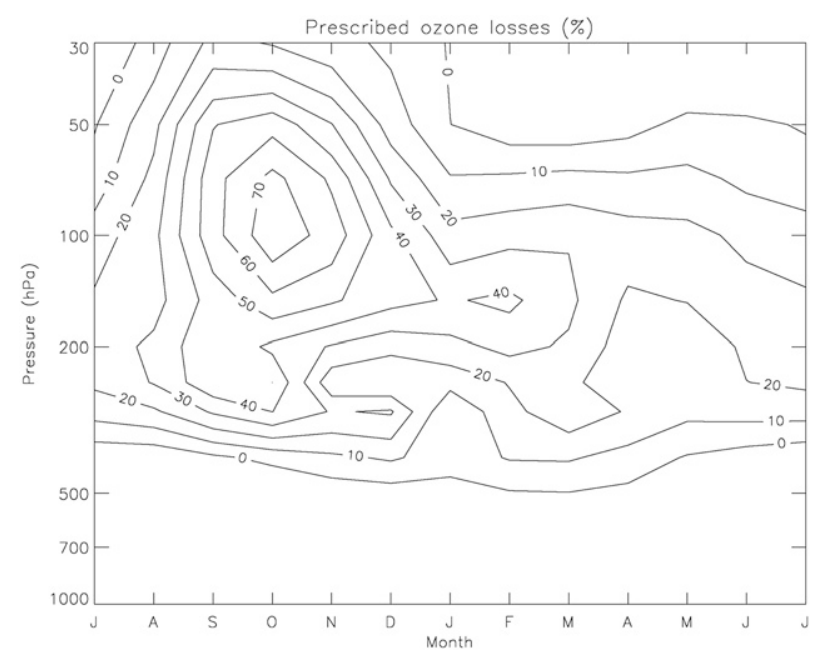

FIG. 1. Time-height cross section of the difference in prescribed ozone between the ozone hole and preozone hole climatologies at $70^{\circ} \mathrm{S}$. Contour interval is $10 \%$. Tick marks on the ordinate axis denote the middle of the respective month.

\section{b. Data}

The 40-yr European Centre for Medium-Range Weather Forecasts (ECMWF) Re-Analysis (ERA-40) data cover the period from mid-1957 to mid-2002 (Uppala et al. 2005). Only data from 1979 to 2001 are employed in this study, which are representative of the actual impact of ozone depletion as the size of the ozone hole increased steadily during this period (Huck et al. 2007). Six-hourly fields of temperature and winds were retrieved at a resolution of $2^{\circ} \times 2^{\circ}$ at 17 vertical pressure levels: 1000, 925, $850,700,600,500,400,300,250,200,150,100,70,50,30$, 20 , and $10 \mathrm{hPa}$.

\section{c. The transformed Eulerian mean equation}

The mechanism of wave forcing on the zonally averaged zonal wind can be examined using the ageostrophic form of the TEM equation, defined in Dunkerton et al. (1981), as

$$
\frac{\partial \bar{u}}{\partial t}=\left[f-\frac{(\bar{u} \cos \phi)_{\phi}}{a \cos \phi}\right] \bar{v}^{*}-\bar{u}_{z} \bar{w}^{*}+\frac{1}{\rho_{0} a \cos \phi} \nabla \cdot \mathbf{F}+\bar{G}
$$

where $u$ is the zonal wind component, $t$ is time, $f$ is the Coriolis parameter, $a$ is the radius of the earth, $\phi$ is latitude, $z$ is height, $\rho_{0}$ is the background density, and $G$ is the unresolved forcing. The Eliassen-Palm (EP) wave flux is given as $\mathbf{F}=\left(F_{(\phi)}, F_{(z)}\right)$, with $F_{(\phi)}=\rho_{0} a \cos \phi$ $\left[\bar{u}_{z}\left(\overline{v^{\prime} \theta^{\prime}} / \bar{\theta}_{z}\right)-\overline{u^{\prime} v^{\prime}}\right]$ and $F_{(z)}=\rho_{0} a \cos \phi\left(\left\{f-\left[(\bar{u} \cos \phi)_{\phi}{ }^{\prime}\right.\right.\right.$ $\left.a \cos \phi]\}\left(\overline{v^{\prime} \theta^{\prime}} / \bar{\theta}_{z}\right)-\overline{w^{\prime} u^{\prime}}\right)$, and its divergence is given as $\boldsymbol{\nabla} \cdot \mathbf{F}=(a \cos \phi)^{-1} \partial\left(\cos \phi F_{(\phi)}\right) / \partial \phi+\partial F_{(z)} / \partial z$, where $v$ is 
the meridional wind component, $w$ is the vertical wind component, $\theta$ is the potential temperature, $\overline{v^{\prime} \theta^{\prime}}$ is the horizontal eddy heat flux, and $\overline{u^{\prime} v^{\prime}}$ is the horizontal eddy momentum flux. The residual meridional circulation $\left(\bar{v}^{*}, \bar{w}^{*}\right)$ is given by $\bar{v}^{*}=\bar{v}-\rho_{0}^{-1} \partial\left[\rho_{0}\left(\overline{v^{\prime} \theta^{\prime}} / \bar{\theta}_{z}\right)\right] / \partial z$ and $\bar{w}^{*}=\bar{w}+(a \cos \phi)^{-1} \partial\left[\cos \phi\left({\overline{v^{\prime}} \theta^{\prime} / \bar{\theta}_{z}}_{z}\right)\right] / \partial \phi$. Overbars denote zonal means and primes denote deviations from the zonal mean. Subscripts denote the quantity with respect to which the partial derivative is taken.

The EP flux $\mathbf{F}$ roughly approximates the direction of wave energy propagation. A decelerating force is exerted on $\bar{u}$ wherever $\mathbf{F}$ converges, as identified by the second term on the right-hand side (rhs) of (1), often referred to as wave driving of the zonal mean flow (Edmon et al. 1980; Dunkerton et al. 1981). For example, planetary waves typically break in the stratosphere during wintertime (McIntyre and Palmer 1983), with the resulting convergence of $\mathbf{F}$ causing a deceleration of the eastward polar vortex. This deceleration of the mean zonal flow in the stratosphere is largely counteracted by a mean meridional circulation (MMC), referred to as the Brewer-Dobson circulation, in which air rises in the tropics and then moves poleward and downward (Fig. 2 of Plumb 2002). Using the TEM approach, this circulation is approximated by $\left(\bar{v}^{*}, \bar{w}^{*}\right)$. The first term on the rhs of (1) represents the MMC driving; it is dominated by the term $f \bar{v}^{*}$, such that the poleward branch of the circulation induces a eastward force (i.e., the MMC driving opposes the wave driving). Conversely, the returning equatorward branch of the MMC induces a westward force at lower altitudes. The sum of the wave driving and MMC driving gives the total zonal flow acceleration (i.e., the net driving) that would be produced in the absence of unresolved smaller-scale forcing. The left-hand side of (1) is the acceleration of the zonal wind (i.e., the zonal wind tendency $\partial \bar{u} / \partial t$ ). Equation (1) is additionally weighted by surface area, using the cosine of the latitude.

\section{d. Maximum Eady growth rate}

A common measure of baroclinicity is the maximum Eady growth rate given by

$$
\sigma_{E}=(-1) \times 0.31 \frac{g}{N \bar{T}} \frac{\partial \bar{T}}{\partial y},
$$

where $g$ is the gravitational acceleration, $N$ is the buoyancy frequency, $T$ is temperature, and $y$ is the meridional direction (Hoskins and Valdes 1990; Yin 2005). We have omitted the $(-1)$ term to give positive values in the $\mathrm{SH}$ midlatitudes. This parameter is estimated for a mass-weighted average of the troposphere (850$300 \mathrm{hPa}$ ), with levels in the boundary layer omitted (below $850 \mathrm{hPa}$ ) (Hoskins and Valdes 1990). Similar results were obtained with $\sigma_{E}$ computed just above the boundary layer (at $700 \mathrm{hPa}$ ).

\section{e. Refractive index}

We will account for the differences in wave propagation by computing the square of the quasigeostrophic refractive index, defined as (Chen and Robinson 1992)

$$
n_{s}^{2}=\frac{\bar{q}_{\phi}}{a(\bar{u}-c)}-\left(\frac{s}{a \cos \phi}\right)^{2}-\left(\frac{f}{2 N H}\right)^{2},
$$

where

$$
\bar{q}_{\phi}=\frac{2 \Omega}{a} \cos \phi-\frac{1}{a^{2}}\left[\frac{(\bar{u} \cos \phi)_{\phi}}{\cos \phi}\right]_{\phi}-\frac{f^{2}}{\rho_{0}}\left(\rho_{0} \frac{\bar{u}_{z}}{N^{2}}\right)_{z}
$$

is the meridional gradient of potential vorticity, $c$ is the wave zonal phase speed, $s$ is the zonal wavenumber, $H$ is the scale height of the atmosphere, and $\Omega$ is the angular velocity of the earth. Linear wave theory predicts that wave activity or EP flux trajectories can propagate in regions of positive $n_{s}^{2}$ but not in regions where $n_{s}^{2}$ is negative, and will tend to follow the gradient of $n_{s}^{2}$ toward regions of refraction maximum. Here, this index is used to understand differences in the propagation of planetary waves in the lower stratosphere and synopticscale waves in the upper troposphere. It is noted that the assumption of linearity is probably violated for planetary waves (i.e., that the waves are of small amplitude and slowly varying in time). However, even when these conditions do not strictly apply the refractive index has still been shown to be a useful qualitative measure of wave propagation (e.g., Randel 1988; Chen and Robinson 1992; Limpasuvan and Hartmann 2000; Simpson et al. 2009).

\section{f. Statistical significance}

For statistical significance testing of the differences between the two 24-yr model runs, $p$ values are shown based on a one-sided Student's $t$ test. We assume 24 degrees of freedom. This is a valid assumption since the atmosphere-only runs were forced with annually repeating boundary conditions and would therefore not be expected to show any significant memory from year to year. Consistent with this, we found no clear pattern of year-to-year correlations in November-December zonal wind (not shown). The $p$ values of contoured values are shown in our figures by shading, with light (dark) shading denoting regions where $p \leq 0.1(\leq 0.05)$. The $p$ values of vectors shown in our figures are indicated by thick (thin) vectors denoting $p \leq 0.05(>0.05)$. 

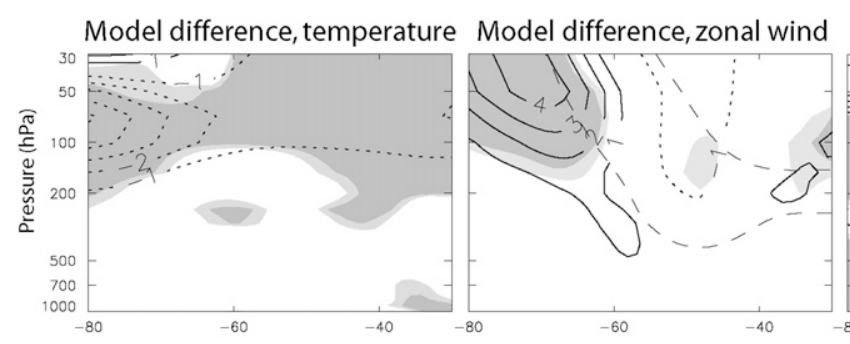

ERA40 trend, temperature
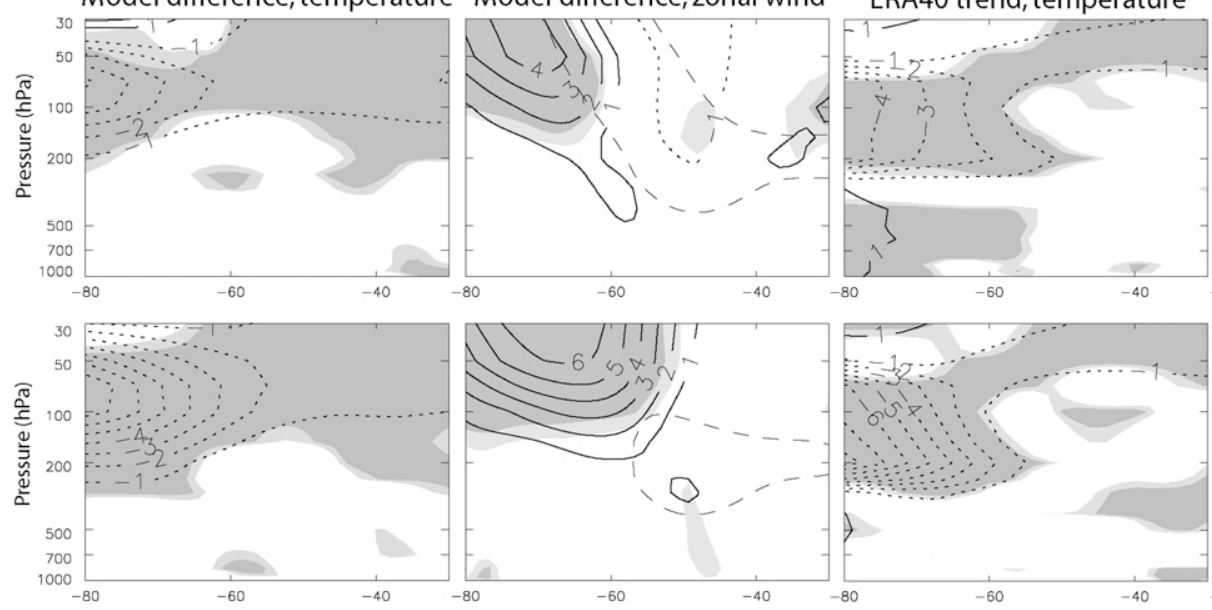

ERA40 trend, zonal wind
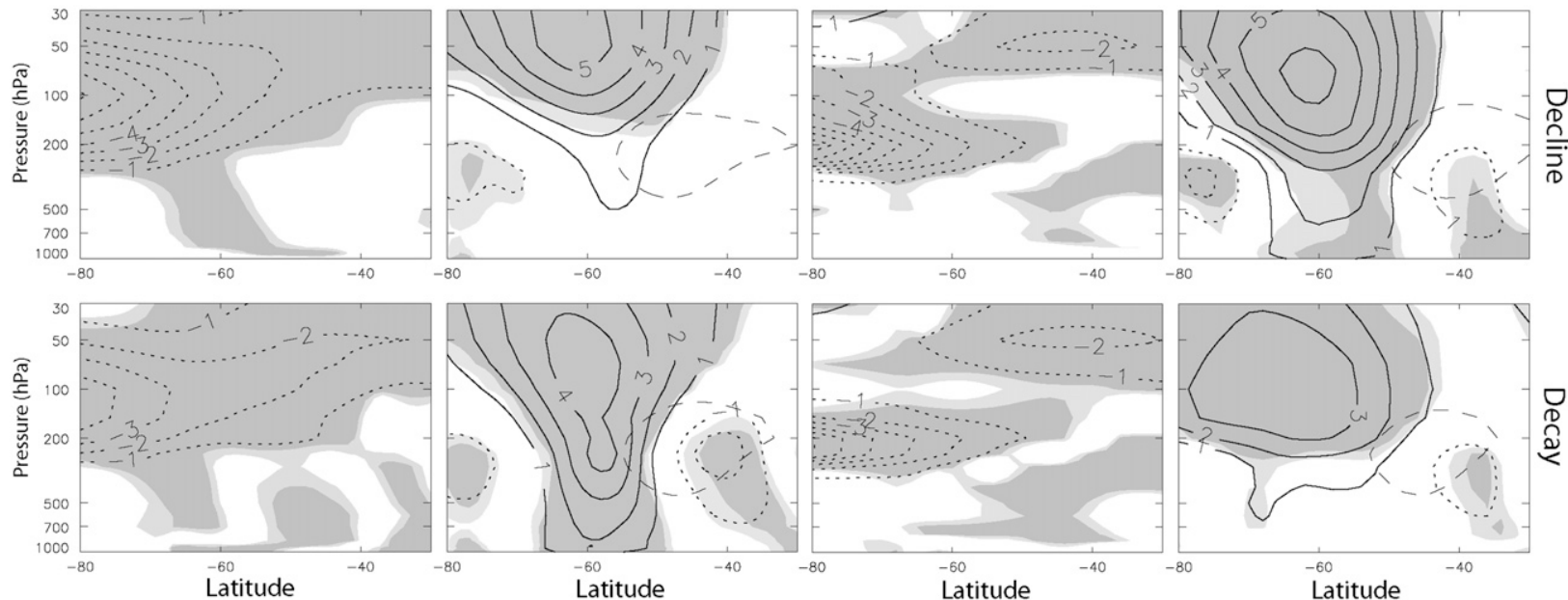

FIG. 2. Latitude-height cross sections of (first and second columns) the simulated and (third and fourth columns) the observed changes of zonal mean temperature and zonal wind for (first row) onset (2-16 Nov), (second row) growth (17 Nov-1 Dec), (third row) decline (2-16 Dec) and (fourth row) decay (17 Dec-1 Jan) stages. Simulated changes are the 24-yr averaged differences between the ozone hole and preozone hole model runs. Observed changes are ERA-40 23-yr linear trends (1979-2001). The contour intervals for the simulated (observed) changes are $1 \mathrm{~K}\left(1 \mathrm{~K} \mathrm{decade}{ }^{-1}\right)$ for temperature and $1 \mathrm{~m} \mathrm{~s}^{-1}\left(1 \mathrm{~m} \mathrm{~s}^{-1}\right.$ decade $\left.^{-1}\right)$ for zonal wind. Dotted lines indicate negative values and the zero contour is omitted. Shading denotes regions where $p$ values of the simulated differences are $\leq 0.1$ (light shading) and $\leq 0.05$ (dark shading). An indication of the latitudinal position of the midlatitude jet's climatological state is given by displaying the simulated (observed) average zonal mean zonal wind at $25 \mathrm{~m} \mathrm{~s}^{-1}$ for the 24-yr preozone hole model run (ERA-40 data for the period 1979-2001) as the dashed contour.

\section{Results}

Figure 2 shows that the life cycle of the zonal wind and temperature model differences shown in the first and second columns displays a strong qualitative similarity to the ERA-40 23-yr linear trends (1979-2001) shown in the third and fourth columns. During the onset stage colder temperatures and stronger eastward winds appear at high latitudes above $200 \mathrm{hPa}$ (i.e., a strengthening of the polar vortex). During the growth stage the colder temperatures and eastward winds strengthen and descend down to the tropopause (which has a height of about $300 \mathrm{hPa}$ at high latitudes). During the decline stage the changes in the stratospheric flow propagate downward into the troposphere, although the strengthening of the tropospheric winds is significantly narrower. Also apparent during this stage is a weakening of the colder temperatures and strengthened eastward winds in the lower stratosphere. The ERA-40 trends show the downward descent reaching the surface, forming part of a dipole pattern in the troposphere with eastward winds at around $60^{\circ} \mathrm{S}$ and westward winds at around $40^{\circ} \mathrm{S}$. The westward winds coincide with the climatological maximum, suggesting that a poleward shift of the tropospheric midlatitude jet has occurred, consistent with the SAM positive phase. 

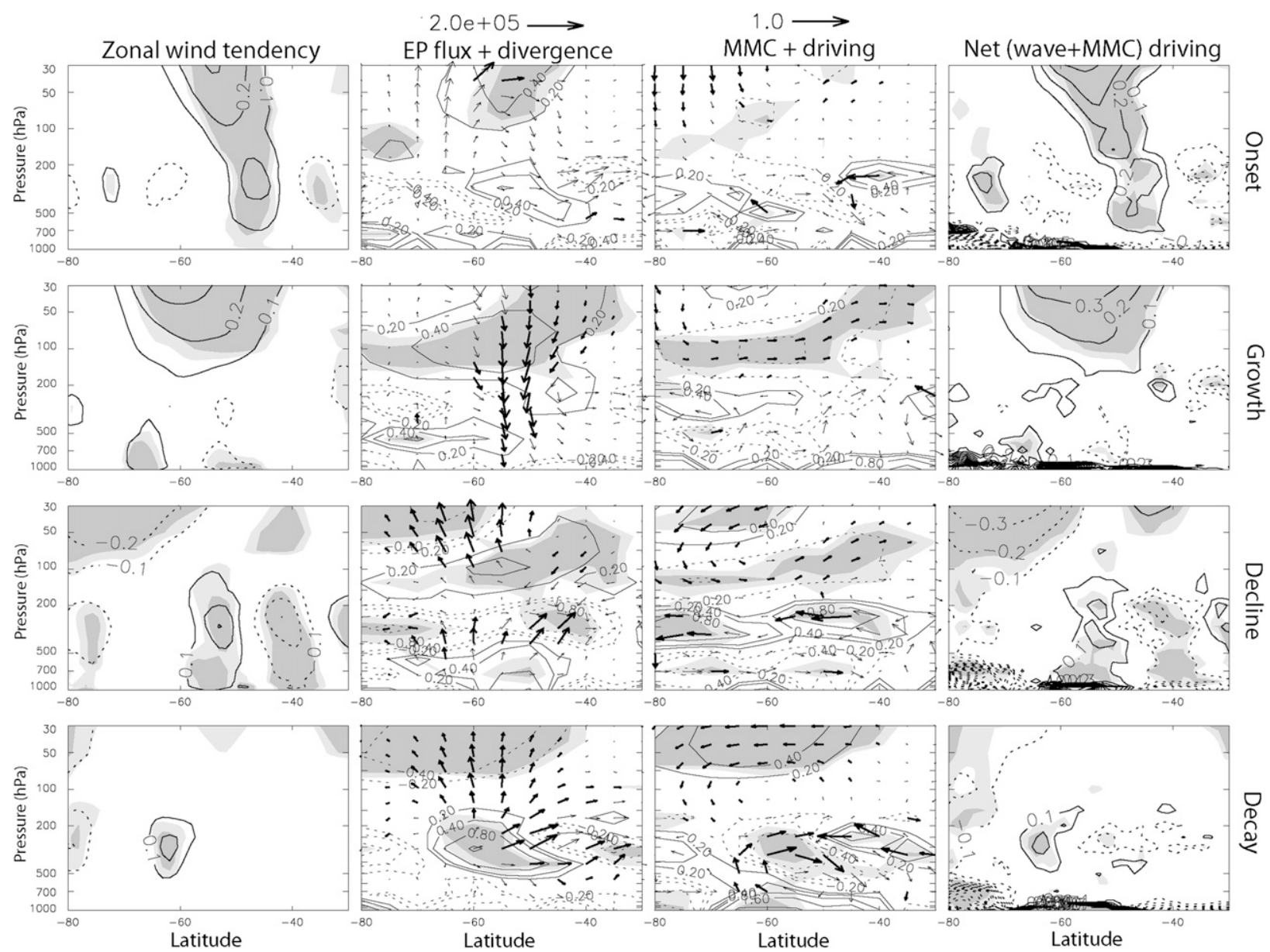

FIG. 3. As in Fig. 2, but showing the simulated changes of (first column) (actual) zonal wind tendency, (second column) wave driving, (third column) MMC driving, and (fourth column) net driving. The contours for the zonal wind tendency and net driving changes are $\pm 0.1,0.2$, $0.3, \ldots \mathrm{m} \mathrm{s}^{-1}$ day $^{-1}$. The contours for the wave driving and MMC driving changes are $\pm 0.2,0.4,0.8,1.6, \ldots \mathrm{m} \mathrm{s}^{-1}$ day $^{-1}$. Dashed lines indicate negative values (westward acceleration) and the zero contour is omitted. Shading denotes regions where the $p$ values of the changes are $\leq 0.1$ (light shading) and $\leq 0.05$ (dark shading). Simulated changes of the EP flux vectors (arrows with reference vector $2.0 \times 10^{5} \mathrm{~m}^{3} \mathrm{~s}^{-2}$ ) and MMC (arrows with reference vector $1.0 \mathrm{~mm} \mathrm{~s}^{-1}$ ) are superimposed on the wave driving and MMC driving plots, respectively. Thick arrows indicate vector differences with $p$ values $\leq 0.05$.

For the model difference results, this response does not fully develop until the decay stage. During the decay stage the zonal wind changes in the troposphere show persistence, in contrast to further weakening of the lowerstratospheric circulation. In fact, both the model and observed changes show the poleward shift of the tropospheric jet persisting into much of January (not shown). The marked similarity between the model and the observed changes 1) confirms that the strengthening of the circumpolar winds from the stratosphere down to the surface over recent decades is primarily in response to ozone depletion, and 2) suggests that investigation of the dynamical characteristics of the model changes will provide some insight into the actual mechanisms involved.

Figure 3 shows model differences of the actual zonal wind tendency $\partial \bar{u} / \partial t$, the wave driving, the MMC driving, and the net driving for the onset, growth, decline, and decay stages. Superimposed difference vectors display the EP flux and the MMC. To compensate for the exaggerated vertical axis in the figures, the horizontal component of the vectors is scaled by a factor $\alpha=$ 0.00441 , which is equal to the vertical distance $(\mathrm{m})$ divided by the horizontal distance spanned by the figures (Palmer 1981). Figure 4 shows model differences of the EP flux and its divergence divided into contributions from planetary waves (i.e., wavenumbers 1-4) and synoptic waves (i.e., wavenumbers 5 and higher) for the various stages. Figure 4 also shows model differences of the tropospheric maximum Eady growth rate. To diagnose the wave driving further, Fig. 5 subdivides the planetary- and synoptic-scale EP flux divergences into their horizontal and vertical components. 

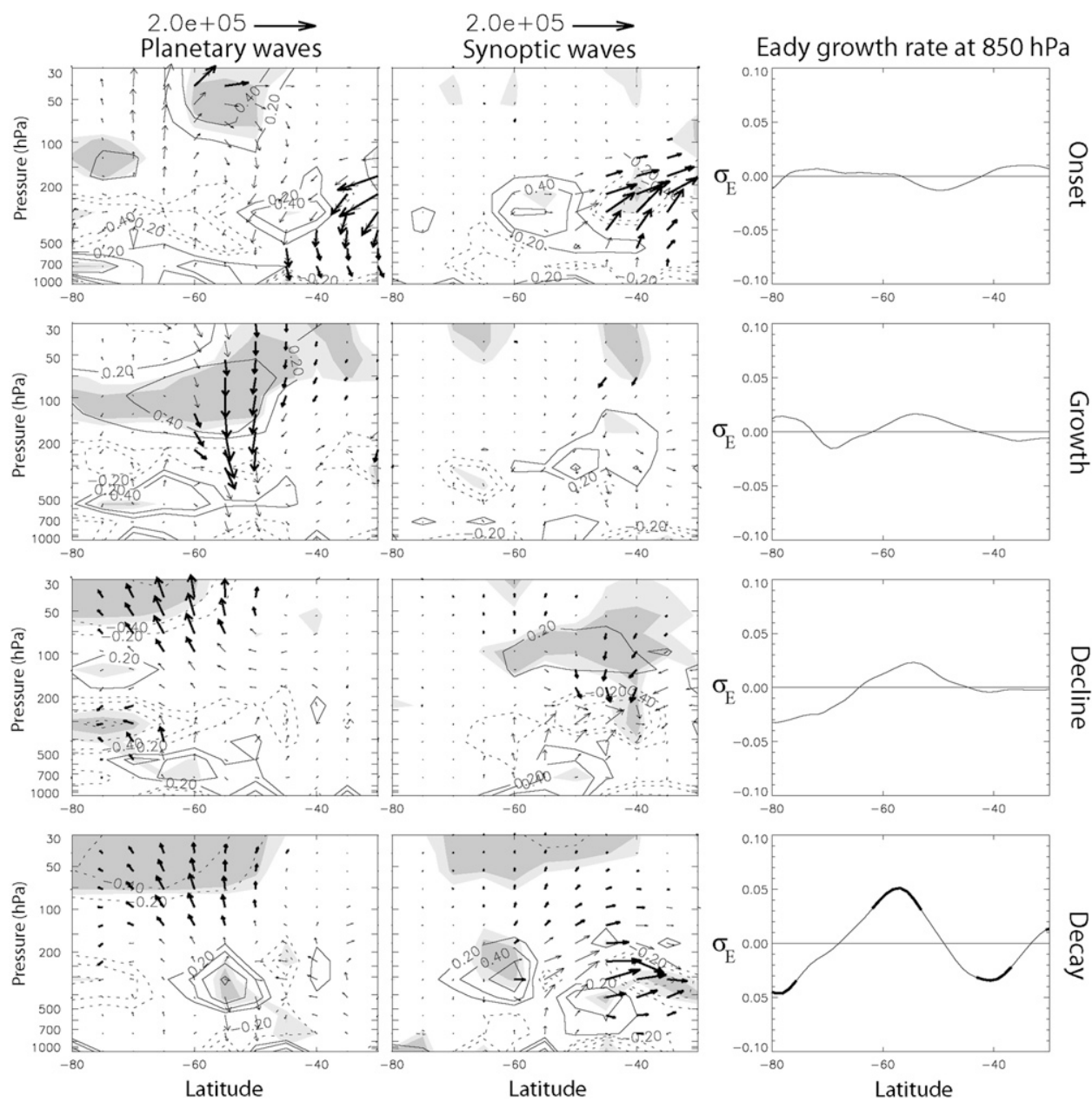

FIG. 4. As in Fig. 2, but showing the simulated changes of (left) planetary-scale and (middle) synopticscale wave driving. The contours for the changes in wave driving are $\pm 0.2,0.4,0.8, \ldots \mathrm{m} \mathrm{s}^{-1}$ day $^{-1}$. Dashed lines indicate negative values (westward acceleration) and the zero contour is omitted. Shading denotes regions where the $p$ values of the changes are $\leq 0.1$ (light shading) and $\leq 0.05$ (dark shading). Changes in planetary- and synoptic-scale EP flux vectors (arrows with reference vector $2.0 \times 10^{5} \mathrm{~m}^{3} \mathrm{~s}^{-2}$ ) are superimposed on the appropriate wave driving plots. Thick arrows indicate vector differences with $p$ values $\leq 0.05$. (right) The simulated changes in the tropospheric $\sigma_{E}\left(\right.$ day $\left.^{-1}\right)$. Latitudes at which the $p$ values for $\sigma_{E}$ are $\leq 0.05$ are indicated by thick sections of the line.

To understand the changes in both planetary and synoptic-scale wave propagation, Fig. 6 shows model differences of $n_{s}^{2}$ for stationary zonal wave $1(s=1, c=$ $0 \mathrm{~m} \mathrm{~s}^{-1}$ ) and for zonal wave 6 and eastward phase speed of $5 \mathrm{~m} \mathrm{~s}^{-1}\left(s=6, c=5 \mathrm{~m} \mathrm{~s}^{-1}\right)$ for each of the various stages. To aid interpretation of the planetary wave differences, Fig. 6 also shows values for the 24-yr averaged preozone hole and ozone hole runs. For planetary waves, we justify computing $n_{s}^{2}$ based on stationary zonal wave 1 [referred to as $n_{(s=1)}^{2}$ ] as Randel (1988) revealed from EP flux cross sections that this dominated the planetary wave driving throughout the $\mathrm{SH}$ stratosphere during October. However, it is noted that $n_{s}^{2}$ for the other planetary waves that are characterized by nonzero phase speeds may differ substantially from that of $n_{(s=1)}^{2}$, particularly when the zonal flow is relatively weak such as during late spring and early summer. For synoptic waves, we simply chose zonal wave 6 as being representative and used time-longitude lag-correlation diagrams (Randel 1988) to estimate that at $50^{\circ} \mathrm{S}$ and $300 \mathrm{hPa}$ (i.e., in the upper troposphere) they exhibited an eastward phase speed of about $5 \mathrm{~m} \mathrm{~s}^{-1}$ over the final two stages. Note that the method of Randel (1988), in which the zonal phase speed is indicated by the slope in time of the maximum negative or positive correlation coefficients, was unable to determine with any certainty whether the wave- 6 phase speed was appreciably different between the preozone hole and ozone hole runs. 

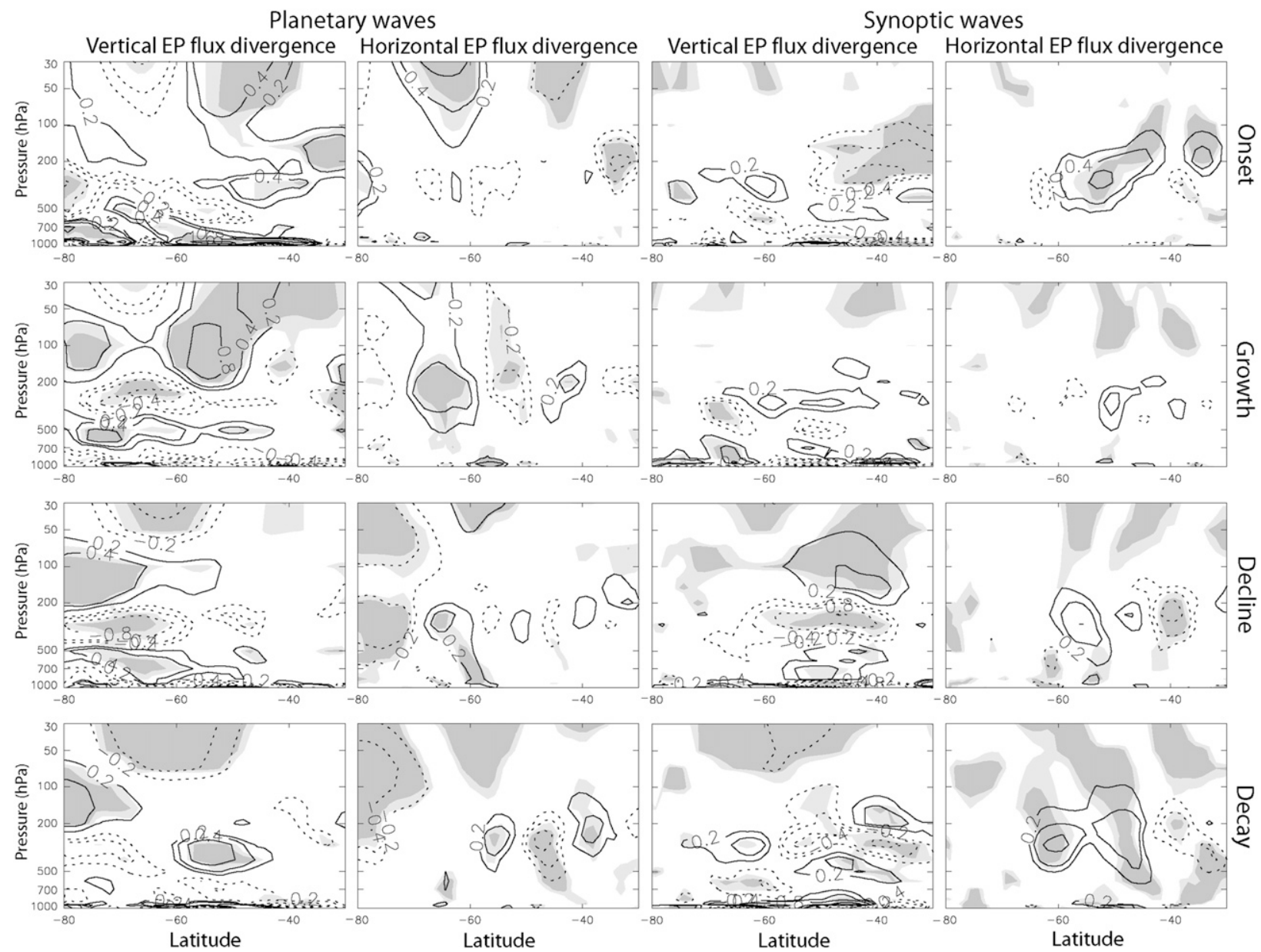

FIG. 5. As in Fig. 2, but showing the simulated changes of (first and second columns) planetary-scale and (third and fourth columns) synoptic-scale horizontal and vertical components of the EP flux divergence. The contours are $\pm 0.2,0.4,0.8,1.6, \ldots \mathrm{m} \mathrm{s}^{-1} \mathrm{day}^{-1}$. Shading denotes regions where the $p$ values of the changes are $\leq 0.1$ (light shading) and $\leq 0.05$ (dark shading).

Examination of the main features of these results, and their similarity to the dynamical mechanisms outlined above, now follows. The model differences in the lower stratosphere will be examined first, followed by the troposphere.

\section{a. Lower-stratosphere model differences}

During the onset stage the model difference of the actual zonal wind tendency shows eastward acceleration in the lower stratosphere, consistent with the strengthening of the zonal wind (Fig. 2). The upward (downward) EP flux poleward (equatorward) of $60^{\circ} \mathrm{S}$ indicates a strengthening (weakening) of wave activity in the lower stratosphere (Fig. 3) that is almost entirely due to planetary waves (Fig. 4). The accompanying difference in wave driving is eastward, consistent with the actual zonal wind tendency, and drives an equatorward MMC in the lower stratosphere. The associated westward acceleration partially offsets the eastward acceleration of the wave driving. The resulting net eastward driving in the lower stratosphere agrees in pattern but is slightly larger in amplitude (by about $0.1 \mathrm{~m} \mathrm{~s}^{-1}$ day $^{-1}$ ) compared to the actual zonal wind tendency (Fig. 3).

During this stage the differences in $n_{(s=1)}^{2}$ equatorward of $60^{\circ} \mathrm{S}$ shows a reduction in the lower stratosphere and an increase below it (Fig. 6), consistent with the anomalous propagation of planetary wave activity following the gradient of $n_{(s=1)}^{2}$ toward regions of refraction maximum. Poleward of $60^{\circ} \mathrm{S}$ shows an increase of $n_{(s=1)}^{2}$ in the lower stratosphere, consistent with the anomalous propagation of planetary wave activity toward regions of positive $n_{(s=1)}^{2}$. Detailed examination (not shown) shows that these differences in $n_{(s=1)}^{2}$ are mainly accounted for by the first term on the rhs of (3) involving $\bar{q}_{\phi} / a(\bar{u}-c)$ (i.e., the meridional gradient of potential vorticity and the mean background wind). Following Simpson et al. (2009) the differences in this term can be expanded in terms of the contribution from the changes in $\bar{q}_{\phi}$ only 

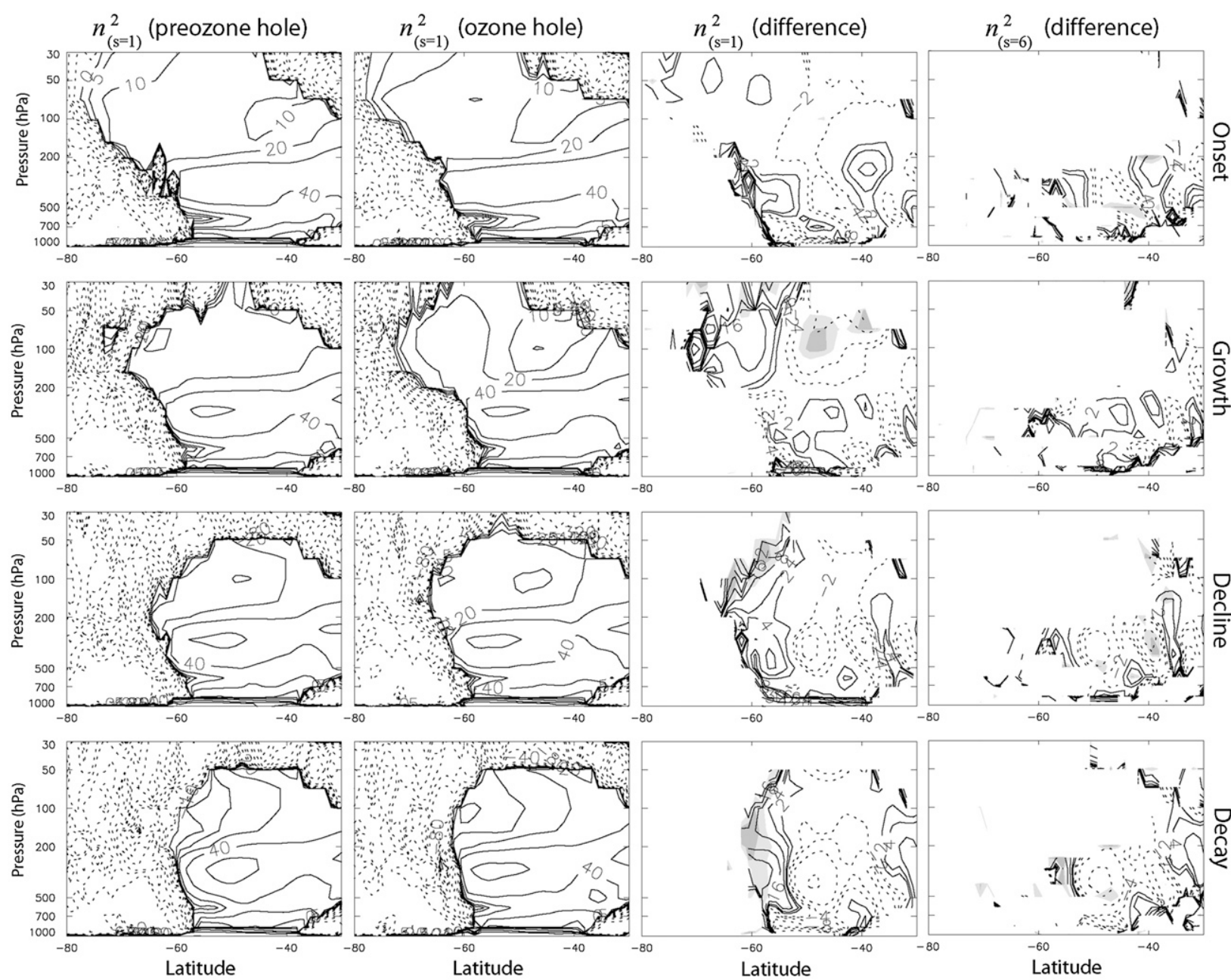

FIG. 6. As in Fig. 2, but showing the simulated changes of the square of refractive index for (third column) $n_{(s=1)}^{2}$ (wave 1, stationary) and (fourth column) $n_{(s=6)}^{2}$ (wave 6, eastward phase speed of about $5 \mathrm{~m} \mathrm{~s}^{-1}$ ). Also shown are the 24-yr averaged $n_{(s=1)}^{2}$ for (first column) preozone hole and (second column) ozone hole runs to aid interpretation of the planetary wave differences. Contours are $\pm 5,10,20,40,80$ for the long-term averages and intervals are 2 for their difference. Dashed lines indicate negative values. The statistical significance has been shown for the differences, with shading denoting regions where $p$ values are $\leq 0.1$ (light shading) and $\leq 0.05$ (dark shading). Note that regions where either the preozone hole or ozone hole $n_{s}^{2}$ values are negative are masked in the corresponding difference fields.

[by calculating the differences in $\bar{q}_{\phi} / a(\bar{u}-c)$ using the preozone hole values of $\bar{u}$, the changes in $\bar{u}$ only [by calculating the differences in $\bar{q}_{\phi} / a(\bar{u}-c)$ using the preozone hole values of $\bar{q}_{\phi}$ ], and a small nonlinear residual. Comparison between these terms showed that most of the differences in $\bar{q}_{\phi} / a(\bar{u}-c)$ are explained by the changes in $\bar{q}_{\phi}$. Further examination of the rhs of (4) showed that the differences in $\bar{q}_{\phi}$ are due to both the second term (which is influenced by the meridional curvature of the zonal wind) and the third term (which is influenced by the vertical curvature of the vertical shear and vertical curvature of the vertical temperature gradient).

During the growth stage the change in eastward zonal wind tendency strengthens in the lower stratosphere
(Fig. 3), in agreement with the strengthening of the zonal wind down to the tropopause (Fig. 2). The strengthening of the anomalous downward planetary wave EP flux causes both 1) increased EP flux divergence and associated eastward wave driving throughout the lower stratosphere, consistent with the actual zonal wind tendency, and 2) increased equatorward MMC and associated westward acceleration (Figs. 3 and 4). The downward propagation is clearly being driven by the vertical component of the planetary wave EP flux divergence (Fig. 5). The resulting net eastward driving in the lower stratosphere agrees broadly in amplitude and pattern with the actual zonal wind tendency (Fig. 3).

During this stage the differences in $n_{(s=1)}^{2}$ equatorward of around $55^{\circ} \mathrm{S}$ show a further reduction in the lower 
stratosphere (Fig. 6), consistent with a strengthening of its downward gradient and with the increase in anomalous downward planetary wave EP flux. Detailed examination (not shown) shows that these differences in $n_{(s=1)}^{2}$ are again accounted for by the term $\bar{q}_{\phi} / a(\bar{u}-c)$, and that the differences in this term are explained by changes in $\bar{q}_{\phi}$. Further examination of the rhs of (4) showed that both the second and third terms contributed to the differences in $\bar{q}_{\phi}$.

During the decline stage the zonal wind tendency change reverses to westward in the lower stratosphere (Fig. 3), in agreement with the weakening of the strengthened eastward winds (Fig. 2). The anomalous EP flux is upward and slightly poleward, dominated by planetary waves, and indicate a strengthening of wave activity in the lower stratosphere above approximately $100 \mathrm{hPa}$. This causes increased EP flux convergence and associated westward wave driving in a region approximately poleward of $60^{\circ} \mathrm{S}$ and above $100 \mathrm{hPa}$, and increased EP flux divergence and associated eastward wave driving in a broad region below (Figs. 3 and 4). These changes are largely driven by the vertical component of the planetary wave EP flux divergence (Fig. 5). This difference in wave forcing drives a poleward (equatorward) MMC in the region of convergence (divergence). The resulting net driving is westward and agrees in pattern but is slightly larger in amplitude (by about $-0.1 \mathrm{~m} \mathrm{~s}^{-1}$ day $^{-1}$ ) compared to the actual zonal wind tendency (Fig. 3).

During this stage the differences in $n_{(s=1)}^{2}$ show an increase in the lower stratosphere at around $60^{\circ} \mathrm{S}$, which slopes upward from high to low latitudes, and a reduction equatorward of this (see Fig. 6, although only the increase is statistically significant), consistent with the anomalous upward and slightly poleward planetary wave EP flux. Detailed examination (not shown) shows that these differences in $n_{(s=1)}^{2}$ are again mainly accounted for by changes in $\bar{q}_{\phi}$, and that the differences in this term are largely explained by the third term on the rhs of (4), which is influenced by the vertical curvature of the vertical shear and vertical curvature of the vertical temperature gradient.

During the decay stage the zonal wind tendency weakens in the lower stratosphere but remains westward (Fig. 3), in agreement with further weakening of the anomalously strong eastward winds (Fig. 2). The EP flux differences are still dominated by planetary waves but are now oriented more upward, slightly reduced, and apparent throughout the lower stratosphere. This results in a significant region of slightly weaker EP flux convergence and poleward MMC (Figs. 3 and 4). These changes are largely driven by the vertical component of the planetary wave EP flux divergence (Fig. 5). The resulting net westward driving in the lower stratosphere agrees in amplitude but is slightly broader in pattern compared to the actual zonal wind tendency (Fig. 3).

During this stage the differences in $n_{(s=1)}^{2}$ are broadly similar to those of the previous stage (Fig. 6) and consistent with the changes in planetary wave EP flux (Fig. 4).

\section{b. Troposphere model differences}

During the decline stage, the changes in the stratospheric flow begin to propagate downward into the troposphere (Fig. 2). The zonal wind tendency changes during this stage show a dipole pattern in the troposphere with eastward acceleration between $50^{\circ}$ and $60^{\circ} \mathrm{S}$ and westward acceleration at around $40^{\circ} \mathrm{S}$ (Fig. 3). Poleward of around $60^{\circ} \mathrm{S}$ the EP flux shows enhanced upward propagation below about $500 \mathrm{hPa}$ followed by poleward propagation between approximately 500 and $200 \mathrm{hPa}$, which are due to planetary waves. This results in a region of EP flux divergence in the lower troposphere and a region of convergence in the upper troposphere at high latitudes (Figs. 3 and 4). This vertical dipole in planetary-scale wave driving is largely due to the vertical component of the EP flux divergence (Fig. 5). By contrast, the anomalous poleward propagation of planetary wave activity gives rise to a horizontal dipole in the upper troposphere, with westward forcing between $70^{\circ}$ and $80^{\circ} \mathrm{S}$ and eastward forcing between $60^{\circ}$ and $70^{\circ} \mathrm{S}$ (Fig. 5). Equatorward of $60^{\circ} \mathrm{S}$, the results show analogous differences due to synoptic waves. However, here the enhanced upward propagation in the troposphere is followed by equatorward propagation in the upper troposphere, although only the total (i.e., synoptic plus planetary-scale) EP flux is statistically significant and not the synoptic wave EP flux. As seen for the planetary waves, these propagation characteristics result in a region of divergence in the lower troposphere and convergence in the upper troposphere, which is largely driven by the vertical component of the EP flux divergence. The horizontal component of the synoptic wave EP flux divergence gives a horizontal dipole in the upper troposphere, with eastward forcing between $50^{\circ}$ and $60^{\circ} \mathrm{S}$ and westward forcing at around $40^{\circ} \mathrm{S}$. It is noted that the enhanced upward synoptic EP fluxes from the lower boundary coincide with latitudes of increased tropospheric $\sigma_{E}$, although the change in $\sigma_{E}$ is not statistically significant (Fig. 4). These changes in wave forcing drives a poleward MMC with associated eastward driving in the upper troposphere, and a returning equatorward MMC with associated westward driving in the lower troposphere. The resulting net driving in the troposphere agrees in amplitude and pattern with the zonal wind tendency (Fig. 3).

During this stage the differences in $n_{(s=6)}^{2}$ in the upper troposphere at around $40^{\circ} \mathrm{S}$ show a horizontal dipole 
resulting in an increased equatorward gradient of $n_{(s=6)}^{2}$ (Fig. 6). This change is consistent with the increased equatorward synoptic wave EP flux. Detailed examination (not shown) shows that these differences in $n_{(s=6)}^{2}$ are mainly accounted for by changes in $\bar{q}_{\phi}$, and that the differences in this term are largely explained by the second term on the rhs of (4), which is influenced by horizontal curvature of the horizontal shear (i.e., barotropic shear).

During the decay stage, the zonal wind tendency changes show eastward acceleration in the troposphere at a latitude slightly poleward of its location during the decline stage (Fig. 3), in agreement with the dipole pattern in the troposphere and consistent with a poleward shift of the midlatitude jet (Fig. 2). A region of westward acceleration also occurs at lower latitudes but is not apparent in the plot as its amplitude is smaller than the minimum contour interval of $0.1 \mathrm{~m} \mathrm{~s}^{-1}$ day $^{-1}$ (Fig. $3)$. The anomalous EP flux shows a region of flux divergence in the middle and upper troposphere, flanked either side by regions of convergence (although only the upward and equatorward EP flux is statistically significant). The change in planetary wave EP flux is not statistically significant, but the associated change in planetary wave EP flux divergence is. They show anomalous downward planetary wave EP flux between $50^{\circ}$ and $60^{\circ} \mathrm{S}$ (Fig. 4), which results in regions of divergence in the upper troposphere and convergence below, and anomalous poleward planetary wave EP flux in the upper troposphere between around $40^{\circ}$ and $60^{\circ} \mathrm{S}$, which results in a tripole of wave forcing (Fig. 5). The changes to the synoptic wave EP flux are similar to those of the previous stage but amplified (although only the anomalous equatorward EP flux is statistically significant). It is noted that the resulting horizontal dipole in the upper troposphere produced by the equatorward synoptic wave EP flux is significantly amplified compared to the previous stage, with the region of eastward forcing considerably strengthened and displaced poleward (Fig. 5). It is also noticed that $\sigma_{E}$ has increased appreciably at around $60^{\circ} \mathrm{S}$ and decreased at around $40^{\circ} \mathrm{S}$, with these changes in baroclinicity matching the dipole structure of the anomalous zonal wind (Fig. 2). The increase in upward synoptic wave EP flux from the lower boundary coincides with the source of enhanced baroclinicity. The resulting net driving in the troposphere shows a dipole pattern which is displaced poleward from its location during the decline stage. The eastward region of net driving agrees in amplitude and pattern with the zonal wind tendency. The westward region of net driving is slightly larger than the zonal wind tendency.

During this stage the differences in $n_{(s=6)}^{2}$ in the upper troposphere at around $40^{\circ} \mathrm{S}$ show the equatorward gradient strengthening, consistent with the amplification of the equatorward synoptic wave EP flux. Detailed examination (not shown) shows again that these differences in $n_{(s=6)}^{2}$ are mainly accounted for by changes in $\bar{q}_{\phi}$, and that the differences in this term are largely explained by the second term on the rhs of (4).

It is noted that the eastward regions of net driving and zonal wind tendency during the decay stage are significant at the $p \leq 0.05$ level. Areas significant at this level would by chance be expected to appear in $1 / 20$ of the total area of the plots, which is approximately the size of the area occupied by these regions. Reinforcement of their importance is, however, provided by the observation that they are dynamically consistent with clearly significant differences in EP flux, wave driving, and MMC driving.

\section{Summary and discussion}

It is found, consistent with other modeling studies (e.g., Gillett and Thompson 2003), that stratospheric ozone depletion results in an anomalous strengthening of the SH circumpolar flow from the lower stratosphere to the surface. The response in our model is broadly in agreement with the observed changes (e.g., Thompson and Solomon 2002), allowing the dynamics of the actual mechanisms involved to be explored by examining those in the model. The results demonstrate that two positive feedback processes operate: one involving a reduction in the upward propagation of planetary waves, which is important for the downward propagation in the stratosphere, and the second involving changes to the synopticscale eddy fluxes of heat and momentum responsible for driving the tropospheric annular modes, which is important for the poleward displacement of the midlatitude jet. It is noted that the sum of the zonal wind forcings induced individually by these processes is in broad quantitative agreement with the actual zonal wind tendency.

Taking the stratospheric response first, our findings are suggestive that the (radiative) strengthening of the vortex as a result of ozone depletion conditions the vortex so that, during the onset stage of the seasonal evolution of the ozone hole, fewer planetary waves propagate up from the troposphere. This reduction of wave activity in the stratosphere causes a decrease in the wave-driven deceleration of the polar vortex, resulting in its acceleration. This initiates a feedback in which fewer planetary waves propagate up at successively lower altitudes, further drawing the decrease in wavedriven deceleration and the associated strengthened winds downward toward the tropopause (during the growth stage). This change is primarily driven by the 
vertical convergence of EP flux. The positive feedback between the strengthened vortex and the propagation of planetary waves is consistent with the findings of Chen and Robinson (1992), who showed that increased vertical shear in the lower stratosphere and tropopause region reduces how much planetary wave activity in the troposphere can propagate into the stratosphere. The downward propagation of zonal mean wind anomalies in the stratosphere by the vertical convergence of EP flux has been shown previously (Kuroda and Kodera 1999; Baldwin and Dunkerton 2001; Christiansen 2001).

It should be noted that the strengthening of the anomalous colder temperatures in the polar lower stratosphere during the onset and growth stages (Fig. 2) is inconsistent with the stronger wave-driven downwelling and associated adiabatic warming over the pole (Fig. 3). This implies that the direct radiative cooling of the stratosphere caused by ozone depletion still dominates the temperature tendency during these stages, and by extension that changes in the actual zonal wind tendency are the result of both wave and radiative driving (i.e., we do not unambiguously suggest that the changes in the lower stratosphere are entirely the result of wave driving). However, the contributions of radiative versus dynamical processes to the temperature differences were not examined here but will be the subject of future study. Furthermore, the increase in downwelling over the pole that largely characterizes these two stages appears to be inconsistent with the eastward wave driving in the lower stratosphere, which would typically be associated with a weakening of the Brewer-Dobson circulation (i.e., a reduction in downwelling over the pole). This suggests that increased wave driving at levels above $30 \mathrm{hPa}$ - which following the "downward control" principle (Haynes et al. 1991) controls the circulation lower down-is important. This discrepancy will also be the subject of future study. Moreover, the residual between the actual zonal wind tendency and the net driving suggests that a component of westward (negative) acceleration of the vortex is required in addition to the net driving. It is plausible that this missing driver is due to unresolved smaller-scale forcing, as identified by the third term on the rhs of (1). Here, a westward acceleration could be explained by an increase in wave driving resulting from increased critical level filtering of gravity waves in response to the stronger vortex winds (e.g., Manzini et al. 2003).

Turning to the tropospheric response, our findings are suggestive of enhanced baroclinic activity (during the decay and decline stages), as indicated by upward synoptic wave EP flux anomalies (increased poleward heat flux), followed by an equatorward propagation of the eddy activity (increased poleward momentum flux) in the upper troposphere. This is consistent with the eddyzonal flow interaction required to drive the anomalous midlatitude jet poleward, associated with the positive phase of the SAM (e.g., Karoly 1990; Robinson 1991). A feedback mechanism is evident whereby an increase in low-level baroclinicity, and the subsequent generation of baroclinic activity, is collocated with the anomalous midlatitude jet, inducing enhanced eddy-zonal flow interaction that acts to reinforce the jet and thus preserve the eastward shear (during the decay stage) (e.g., Robinson 1996, 2000; Lorenz and Hartmann 2001). As the dipole pattern in anomalous zonal wind strengthens because of the poleward shift of the midlatitude jet, the strengthening barotropic shear increasingly refracts the wave activity equatorward, which results in poleward momentum transport (Simpson et al. 2009, 2012). The patterns in the eddy fluxes are consistent with the LC1 baroclinic life cycle found when anticyclonic shear dominates, which is characterized by anomalously strong anticyclonic Rossby wave breaking on the equatorward side of the midlatitude jet (Thorncroft et al. 1993). Additionally, the anomalous midlatitude jet is maintained against surface friction near the surface by a poleward directed MMC and its accompanying eastward acceleration (during the decay stage) (Hartmann and Lo 1998). It is noted that the suggested mechanism is consistent with the following observed summer trends during recent decades: 1) an increase in baroclinicity at high latitudes (Castanheira et al. 2009) and 2) an increase in anticyclonic Rossby wave breaking on the equatorward side of the midlatitude jet (Wang and Magnusdottir 2011). The poleward displacement of the tropospheric midlatitude jet by changes to the eddy fluxes of heat and momentum was suggested previously as the method by which the effects of stratospheric ozone depletion can influence the $\mathrm{SH}$ tropospheric circulation (Hartmann et al. 2000; Thompson et al. 2011).

The results suggest that the vertical dipole in planetary wave driving at high latitudes provides the method by which the strengthened stratospheric vortex is initially coupled to the tropospheric response (during the decline stage). Friction damps the winds at the surface so that the eastward planetary wave acceleration associated with the divergence increases the vertical shear in the zonal wind which enhances the low-level baroclinicity. The importance of planetary waves in this role was also suggested by Song and Robinson (2004) (compare Fig. 4c of Song and Robinson with the planetary wave driving during the decline stage in Figs. 4 and 5 herein). A plausible explanation for this anomalous planetary wave EP flux is that the strengthening of the lower-stratospheric wind results in the trapping of planetary wave activity in the troposphere, 

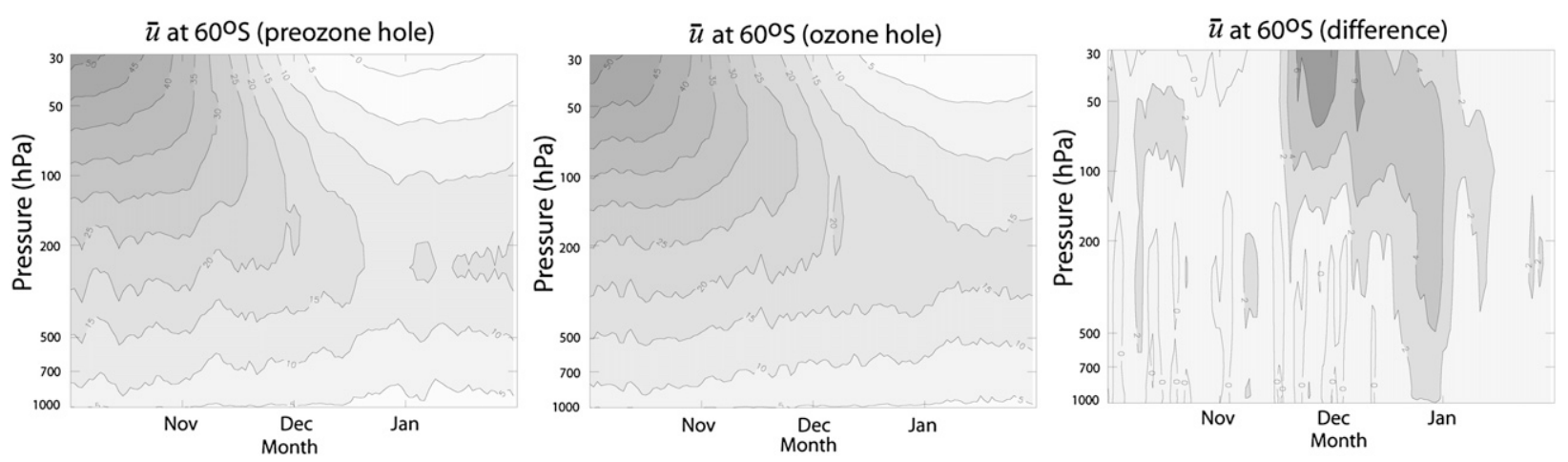

FIG. 7. Time-height cross section of the simulated 24 -yr averaged zonal mean zonal wind at $60^{\circ} \mathrm{S}$ for (left) the preozone hole model run and (middle) the ozone hole model run, and (right) their difference. Contour interval is $5 \mathrm{~m} \mathrm{~s}^{-1}$. Tick marks on the ordinate axis denote the beginning of the respective months. The statistical significance is not shown for these changes as it is demonstrated in Fig. 2 that differences in zonal mean zonal wind are significant at the $p \leq 0.05$ level.

as suggested by Chen and Robinson (1992) and Song and Robinson (2004). An alternate explanation is that during the period of ozone depletion, the anomalous strong and long-lasting (delayed breakup) stratospheric vortex results in the increased reflection of planetary wave activity by the stratosphere back down to the troposphere (Perlwitz and Harnik 2003; Shaw et al. 2011).

Song and Robinson (2004) and Kushner and Polvani (2004) suggest that the strengthened stratospheric vortex could couple to the troposphere by inducing MMC circulations that extend down to the surface. In turn, the changes in vertical motion can increase the local near-surface meridional temperature gradient and thus trigger the transient-eddy feedback responsible for anomalous annular mode variability. However, it was difficult to determine from our results the importance of this response.

Our results suggest that the equatorward propagation of synoptic waves in the upper troposphere, which is important in producing the poleward shift of the midlatitude jet, results from the changes in meridional shear altering the refractive index (Simpson et al. 2009, 2012). By contrast, Chen and Held (2007) argued that the strengthening of the lower-stratospheric wind increases the eastward phase speed of the synoptic eddies and hence the latitude of subtropical wave breaking shifts the pattern of eddy momentum flux convergence poleward. However, our analysis using the time-longitude lag-correlation diagrams of Randel (1988) was not sufficiently accurate to determine with any certainty whether the zonal phase speed of synoptic-scale waves increased between the preozone hole and ozone hole simulations.

The results also explain the delay in the breakup of the strengthened lower-stratospheric vortex. The increase in the propagation of planetary waves into the stratosphere during the decline and decay stages causes an increase in the wave-driven deceleration of the polar vortex [also shown by McLandress et al. (2010)] responsible for the weakening of the strengthened vortex and its subsequent delayed breakup. In this case, the weakening of the anomalously cold temperatures (Fig. 2) is consistent with the stronger wave-driven downwelling and associated adiabatic warming over the pole (Fig. 3). It should be noted that the residual between the actual zonal wind tendency and the net driving during these stages suggests that a component of eastward (positive) acceleration of the vortex is required in addition to the net driving. This is consistent with radiative cooling of the polar stratosphere still being important (albeit diminished).

It is noted that the local maximum in refractive index difference in the lower stratosphere during the decline and decay stages is consistent with a larger refractive index "cavity" (i.e., the region of positive refractive index bounded by a critical layer). This is most apparent by examining the actual refractive index values for the preozone hole and ozone hole simulations (Fig. 6), with the ozone hole simulation showing the cavity extending farther upward and poleward (this is readily apparent at $60^{\circ} \mathrm{S}$ ). This change is due to the later breakup of the polar vortex in the ozone hole simulation (Fig. 7), which represents itself by the delayed descent of zonal-wind jet maximum and a delay in the appearance of westward winds (i.e., an upward shift of the zero-wind line). Perlwitz and Harnik (2003) show that a critical layer is generally located above the jet maximum in the lower stratosphere; that is, it is sensitive to the vertical curvature of $\bar{u}$. Therefore, a consequence of the delayed descent of the jet maximum is a delay in the descent of the critical layer (i.e., a larger refractive index cavity). A key implication of this is that the upward shift in the zero-wind line is not directly responsible for the increase in lower-stratospheric upward planetary wave propagation. 
These findings will facilitate improved understanding of the response to Antarctic ozone depletion (Eyring et al. 2006) and better prediction of future changes in strength of tropospheric westerlies (Son et al. 2008) and of the associated impact on the regional tropospheric $\mathrm{SH}$ climate. These findings also contribute to a better understanding of how stratospheric forcing from other mechanisms, such as changing solar activity (Haigh et al. 2005; Simpson et al. 2009) or changes in water vapor (Maycock et al. 2012), is able to influence the troposphere.

Acknowledgments. The valuable comments of the two anonymous reviewers contributed tremendously to the improvement of the original manuscript. We also thank P. Braesicke, P. Haynes, N. Harnik, J. King, G. Marshall, A. Maycock, M. McIntyre, H. K. Roscoe, J. Scinocca, and J. Turner for valuable discussions on this work. Thanks also to L. Abraham, S. Hardiman, S. Osprey, and P. Telford for help in creating the ozone climatologies. We acknowledge the modeling groups, the Program for Climate Model Diagnosis and Intercomparison (PCMDI), and the WCRP's Working Group on Coupled Modelling (WGCM) for their roles in making available the WCRP CMIP5 multi-model dataset. Support of this dataset is provided by the Office of Science, U.S. Department of Energy.

\section{REFERENCES}

Arblaster, J. M., and G. A. Meehl, 2006: Contributions of external forcings to southern annular mode trends. J. Climate, 19, 28962905.

Baldwin, M. P., and T. J. Dunkerton, 2001: Stratospheric harbingers of anomalous weather regimes. Science, 294, 581-584.

Barnes, E. A., and D. L. Hartmann, 2010: Dynamical feedbacks of the southern annular mode in winter and summer. J. Atmos. Sci., 67, 2320-2330.

Castanheira, J. M., J. A. Añel, C. A. F. Marques, J. C. Antuña, M. L. R. Liberato, L. de la Torre, and L. Gimeno, 2009: Increase of upper troposphere/lower stratosphere wave baroclinicity during the second half of the 20th century. Atmos. Chem. Phys., 9, 9143-9153.

Chen, G., and I. M. Held, 2007: Phase speed spectra and the recent poleward shift of Southern Hemisphere surface westerlies. Geophys. Res. Lett., 34, L21805, doi:10.1029/2007GL031200.

Chen, P., and W. A. Robinson, 1992: Propagation of planetary waves between the troposphere and stratosphere. J. Atmos. Sci., 49, 2533-2545.

Christiansen, B., 2001: Downward propagation of zonal mean zonal wind anomalies from the stratosphere to the troposphere: Model and reanalysis. J. Geophys. Res., 106, 27 307-27 322.

Cionni, I., and Coauthors, 2011: Ozone database in support of CMIP5 simulations: Results and corresponding radiative forcing. Atmos. Chem. Phys. Discuss., 11, 10 875-10 933.

Dunkerton, T., C.-P. F. Hsu, and M. E. McIntyre, 1981: Some Eulerian and Lagrangian diagnostics for a model stratospheric warming. J. Atmos. Sci., 38, 819-843.
Edmon, H. J., B. J. Hoskins, and M. E. McIntyre, 1980: EliassenPalm cross sections for the troposphere. J. Atmos. Sci., 37, 2600-2616.

Eyring, V., and Coauthors, 2006: Assessment of temperature, trace species, and ozone in chemistry-climate model simulations of the recent past. J. Geophys. Res., 111, D22308, doi:10.1029/ 2006JD007327.

Feldstein, S., and S. Lee, 1998: Is the atmospheric zonal index driven by an eddy feedback? J. Atmos. Sci., 55, 3077-3086.

Fogt, R. L., J. Perlwitz, A. J. Monaghan, D. H. Bromwich, J. M. Jones, and G. J. Marshall, 2009: Historical SAM variability. Part II: Twentieth-century variability and trends from reconstructions, observations, and the IPCC AR4 models. J. Climate, 22, 5346-5365.

Gillett, N. P., and D. W. J. Thompson, 2003: Simulation of recent Southern Hemisphere climate change. Science, 302, 273-275.

Graversen, R. G., and B. Christiansen, 2003: Downward propagation from the stratosphere to the troposphere: A comparison of the two hemispheres. J. Geophys. Res., 108, 4780, doi:10.1029/2003JD004077.

Haigh, J. D., and H. R. Roscoe, 2009: The final warming date of the Antarctic polar vortex and influences on its interannual variability. J. Climate, 22, 5809-5819.

- M. Blackburn, and R. Day, 2005: The response of tropospheric circulation to perturbations in lower-stratospheric temperature. J. Climate, 18, 3672-3685.

Hartmann, D. L., 1995: A PV view of zonal flow vacillation. J. Atmos. Sci., 52, 2561-2576.

- , and F. Lo, 1998: Wave-driven zonal flow vacillation in the Southern Hemisphere. J. Atmos. Sci., 55, 1303-1315.

_ J. M. Wallace, V. Limpasuvan, D. W. J. Thompson, and J. R. Holton, 2000: Can ozone depletion and global warming interact to produce rapid climate change? Proc. Natl. Acad. Sci. USA, 97, 1412-1417.

Haynes, P. H., C. J. Marks, M. E. McIntyre, T. G. Shepherd, and K. P. Shine, 1991: On the "downward control" of extratropical diabatic circulations by eddy-induced mean zonal forces. J. Atmos. Sci., 48, 651-678.

Hoskins, B. J., and P. J. Valdes, 1990: On the existence of stormtracks. J. Atmos. Sci., 47, 1854-1864.

Huck, P. E., S. Tilmes, G. E. Bodeker, W. J. Randel, A. J. McDonald, and H. Nakajima, 2007: An improved measure of ozone depletion in the Antarctic stratosphere. J. Geophys. Res., 112, D11104, doi:10.1029/2006JD007860.

Karoly, D. J., 1990: The role of transient eddies in low-frequency zonal variations of the Southern Hemisphere circulation. Tellus, 42A, 41-50.

Karpechko, A. Yu., N. P. Gillet, G. J. Marshall, and A. A. Scaife, 2008: Stratospheric influence on circulation changes in the Southern Hemisphere troposphere in coupled climate models. Geophys. Res. Lett., 35, L20806, doi:10.1029/2008GL035354.

Kuroda, Y., and K. Kodera, 1998: Interannual variability in the troposphere and stratosphere of the Southern Hemisphere winter. J. Geophys. Res., 103, 13 787-13 799.

, and _ 1999: Role of planetary waves in the stratospheretroposphere coupled variability in the Northern Hemisphere winter. Geophys. Res. Lett., 26, 2375-2378.

Kushner, P. J., and L. M. Polvani, 2004: Stratosphere-troposphere coupling in a relatively simple AGCM: The role of eddies. J. Climate, 17, 629-639.

Limpasuvan, V., and D. L. Hartmann, 1999: Eddies and the annular modes of climate variability. Geophys. Res. Lett., 26, $3133-3136$ 
— and - 2000: Wave-maintained annular modes of climate variability. J. Climate, 13, 4414-4429.

— D. W. J. Thompson, and D. L. Hartmann, 2004: The life cycle of the Northern Hemisphere sudden stratospheric warmings. J. Climate, 17, 2548-2596.

Lorenz, D. J., and D. L. Hartmann, 2001: Eddy-zonal flow feedback in the Southern Hemisphere. J. Atmos. Sci., 58, 33123327.

Manzini, E., B. Steil, C. Bruhl, M. A. Giorgetta, and K. Kruger, 2003: A new interactive chemistry-climate model: 2. Sensitivity of the middle atmosphere to ozone depletion and increase in greenhouse gases and implications for recent stratospheric cooling. J. Geophys. Res., 108, 4429, doi: 10.1029/2002JD002977.

Marshall, G. J., 2003: Trends in the southern annular mode from observations and reanalysis. J. Climate, 16, 4134-4143.

Martin, G. M., M. A. Ringer, V. D. Pope, A. Jones, C. Dearden, and T. J. Hinton, 2006: The physical properties of the atmosphere in the new Hadley Centre Global Environmental Model (HadGEM1). Part I: Model description and global climatology. J. Climate, 19, 1274-1301.

Maycock, A. C., M. M. Joshi, K. P. Shine, and A. A. Scaife, 2012: The circulation response to idealized changes in stratospheric water vapor. J. Climate, in press.

McIntyre, M. E., and T. N. Palmer, 1983: Breaking planetary waves in the stratosphere. Nature, 305, 593-600.

McLandress, C., A. I. Jonsson, D. A. Plummer, M, C. Reader, J. F. Scinocca, and T. G. Shepherd, 2010: Separating the dynamical effects of climate change and ozone depletion. Part I: Southern Hemisphere stratosphere. J. Climate, 23, 5002-5020.

Palmer, T. N., 1981: Diagnostic study of a wavenumber-2 stratospheric sudden warming in a transformed Eulerian-mean formalism. J. Atmos. Sci., 38, 844-855.

Perlwitz, J., and N. Harnik, 2003: Observational evidence of a stratospheric influence on the troposphere by planetary wave reflection. J. Climate, 16, 3011-3026.

— S. Pawson, R. L. Fogt, J. E. Nielsen, and W. D. Neff, 2008: Impact of stratospheric ozone recovery on Antarctic climate. Geophys. Res. Lett., 35, L08714, doi:10.1029/2008GL033317.

Plumb, R. A., 2002: Stratospheric transport. J. Meteor. Soc. Japan, 80, 793-809.

Polvani, L. M., and P. J. Kushner, 2002: Tropospheric response to stratospheric perturbations in a relatively simple general circulation model. Geophys. Res. Lett., 29, 1114, doi:10.1029/ 2001 GL014284.

— cursor to extreme stratospheric events and subsequent anomalous surface weather regimes. J. Climate, 17, 3548-3554.

,-- G. J. P. Correa, and S.-W. Son, 2011a: Stratospheric ozone depletion: The main driver of twentieth-century atmospheric circulation changes in the Southern Hemisphere. J. Climate, 24, 795-812.

— M. Previdi, and C. Deser, 2011b: Large cancellation, due to ozone recovery, of future Southern Hemisphere atmospheric circulation trends. Geophys. Res. Lett., 38, L04707, doi:10.1029/2011GL046712.

Randel, W. J., 1988: The seasonal evolution of planetary waves in the Southern Hemisphere stratosphere and troposphere. Quart. J. Roy. Meteor. Soc., 114, 1385-1409.

— stratospheres due to ozone depletion. J. Climate, 12, 1467-1479.

Robinson, W. A., 1991: The dynamics of the zonal index in a simple model of the atmosphere. Tellus, 43A, 295-305.
— 1996: Does eddy feedback sustain variability in the zonal index? J. Atmos. Sci., 53, 3556-3569.

_ 2000: A baroclinic mechanism for the eddy feedback on the zonal index. J. Atmos. Sci., 57, 415-422.

Shaw, T. A., J. Perlwitz, N. Harnik, P. A. Newman, and S. Pawson, 2011: The impact of stratospheric ozone changes on downward wave coupling in the Southern Hemisphere. J. Climate, 24, 4210-4229.

Shindell, D. T., and G. A. Schmidt, 2004: Southern hemisphere climate response to ozone changes and greenhouse gas increases. Geophys. Res. Lett., 31, L18209, doi:10.1029/2004GL020724.

Simpson, I. R., M. Blackburn, and J. D. Haigh, 2009: The role of eddies in driving the tropospheric response to stratospheric heating perturbations. J. Atmos. Sci., 66, 1347-1365.

,$- \ldots$, and —_, 2012: A mechanism for the effect of tropospheric jet structure on the annular mode-like response to stratospheric forcing. J. Atmos. Sci., 69, 2152-2170.

Solomon, S., R. W. Portmann, T. Sasaki, D. J. Hofmann, and D. W. J. Thompson, 2005: Four decades of ozonesonde measurements over Antarctica. J. Geophys. Res., 110, D21311, doi:10.1029/2005JD005917.

Son, S.-W., and Coauthors, 2008: The impact of stratospheric ozone recovery on the Southern Hemisphere westerly jet. Science, 320, 1486-1489.

— N. F. Tandon, L. M. Polvani, and D. W. Waugh, 2009: Ozone hole and Southern Hemisphere climate change. Geophys. Res. Lett., 36, L15705, doi:10.1029/2009GL038671.

__ , and Coauthors, 2010: Impact of stratospheric ozone on Southern Hemisphere circulation change: A multimodel assessment. J. Geophys. Res., 115, D00M07, doi:10.1029/2010JD014271.

Song, Y., and W. A. Robinson, 2004: Dynamical mechanisms for stratospheric influences on the troposphere. J. Atmos. Sci., 61, 1711-1725.

Thompson, D. W. J., and J. M. Wallace, 2000: Annular modes in the extratropical circulation. Part I: Month-to-month variability. J. Climate, 13, 1000-1016.

— Hemisphere climate change. Science, 296, 895-899.

$\longrightarrow$, and - 2005: Recent stratospheric climate trends as evidenced in radiosonde data: Global structure and tropospheric linkages. J. Climate, 18, 4785-4795.

_ M. M. Baldwin, and S. Solomon, 2005: Stratosphere-troposphere coupling in the Southern Hemisphere. J. Atmos. Sci., 62, 708-715.

- S. Solomon, P. J. Kushner, M. H. England, K. M. Grise, and D. J. Karoly, 2011: Signatures of the Antarctic ozone hole in Southern Hemisphere surface climate change. Nat. Geosci., 4, 741-749.

Thorncroft, C. D., B. J. Hoskins, and M. E. McIntyre, 1993: Two paradigms of baroclinic-wave life-cycle behaviour. Quart. J. Roy. Meteor. Soc., 119, 17-55.

Uppala, S. M., and Coauthors, 2005: The ERA-40 Re-Analysis. Quart. J. Roy. Meteor. Soc., 131, 2961-3012.

Wang, Y.-H., and G. Magnusdottir, 2011: Tropospheric Rossby wave breaking and the SAM. J. Climate, 24, 2134-2146.

Wittman, M. A. H., A. J. Charlton, and L. M. Polvani, 2007: The effect of lower stratospheric shear on baroclinic instability. J. Atmos. Sci., 64, 479-496.

Yin, J. H., 2005: A consistent shift of the storm tracks in simulations of 21st century climate. Geophys. Res. Lett., 32, L18701, doi:10.1029/2005GL023684.

Yu, J.-Y., and D. L. Hartmann, 1993: Zonal flow vacillation and eddy forcing in a simple GCM of the atmosphere. J. Atmos. Sci., 50, 3244-3259. 